\title{
Presentation of second primary cancers in young laryngeal carcinoma patients
}

\section{Silen, Suvi}

2019-01-02

Silen , S , Haapaniemi , A , Dickinson , A , Rönn , K \& Mäkitie , A 2019 , ' Presentation of second primary cancers in young laryngeal carcinoma patients ' , Acta Oto-Laryngologica , vol. 139 , no. 1 , pp. 85-89 . https://doi.org/10.1080/00016489.2018.1527037

http://hdl.handle.net/10138/311685

https://doi.org/10.1080/00016489.2018.1527037

unspecified

acceptedVersion

Downloaded from Helda, University of Helsinki institutional repository.

This is an electronic reprint of the original article.

This reprint may differ from the original in pagination and typographic detail.

Please cite the original version. 


\section{Presentation of second primary cancers in young laryngeal carcinoma patients}

Suvi Silén, $\mathrm{MD} \mathrm{PhD*1,2}$; Aaro Haapaniemi, $\mathrm{MD} \mathrm{PhD}^{1 *}$; Amy Dickinson ${ }^{1}, \mathrm{MD}$; Karin Rönn, MD ${ }^{1}$; Antti Mäkitie, MD PhD ${ }^{1,2}$

1 Department of Otorhinolaryngology - Head and Neck Surgery, University of Helsinki and Helsinki University Hospital, Helsinki, Finland ${ }^{2}$ Division of Ear, Nose and Throat Diseases, Department of Clinical Sciences, Intervention and Technology, Karolinska Institutet, Karolinska University Hospital, Stockholm, Sweden

* Equal contribution

This research has received financial support from the Cancer Society of Finland, Finnish Medical Foundation, Finnish-Norwegian Medical Foundation, Jane and Aatos Erkko Foundation, Finnish Research Foundation of Otology, the Helsinki University Hospital Research Fund and the Emil Aaltonen Research Foundation.

Correspondence and reprint requests:

Prof. Antti Mäkitie

Department of Otorhinolaryngology - Head and Neck Surgery,

Helsinki University Hospital, P.O.Box 263, FI-00029 HUS Helsinki, Finland

Tel: +358-50-428 6847, Email: antti.makitie@helsinki.fi

Running title: Second cancers in early onset laryngeal cancer 


\begin{abstract}
Background: Laryngeal squamous cell carcinoma (LSCC) is rare in the young.

Objectives: We characterized the clinical behavior of LSCC and assessed the presentation of second primary tumors (SPCs) in this patient population.

Materials and methods: Data from the Finnish Cancer Registry (FCR) were used to identify an epidemiological series of LSCC patients diagnosed at the age of 40 years or under, during 1953-2012 in Finland. Data regarding primary treatment, survival and SPCs were available. To further characterize the comorbidity and lifestyle factors of young patients with LSCC, institutional data was collected of patients treated at the Helsinki University Hospital during 1967-2012.

Results: We identified 151 patients, with a mean follow-up of 252 months. The 10year overall survival (OS) was $75 \%$ and the disease-specific survival was $84 \%$. SPCs were diagnosed in $26 \%(n=39)$, with a median delay of 28 years. Of the 35 patients in the institutional series from Helsinki, $22(63 \%)$ were current or former smokers. LSCC recurred in $28 \%$ of patients.

Conclusions and Significance: The delay to SPCs in young patients was significantly longer compared with the general LSCC population. As factors underlying this phenomenon cannot be identified by this retrospective study, further studies are warranted.
\end{abstract}

Key words: squamous cell carcinoma, survival, laryngeal cancer, young patients, risk factors, second primary cancer, recurrence 


\section{Introduction}

Laryngeal squamous cell carcinoma (LSCC) forms one of the largest subgroups of head and neck cancer. It typically presents in men (approximately $90 \%$ of patients) in their sixties (1-4). The cumulative carcinogenic burden of smoking and alcohol consumption have been identified as the main risk factors for LSCC: approximately $90 \%$ of patients with LSCC are smokers and the risk of LSCC is 20 -fold in smokers compared with non-smokers. If smoking presents with alcohol consumption, the risk of LSCC is 177-fold (5).

LSCC is rare among young people under the age of 40 years, their occurring in less than $10 \%$ of all LSCC patients $(2,4,6-8)$. Prognostic comparisons between young LSCC patients and the general LSCC population are inconsistent, perhaps due to small sample sizes, different outcome measures and age cut-offs $(6,7,9,10)$. In general, LSCC recurs in up to $30 \%$ of patients $(11,12)$. In addition to recurrence, the risk for second primary cancers (SPCs) in LSCC patients is high, with an incidence exceeding $20 \%$ during the first 10 post-treatment years (3). The incidence of SPCs in the young LSCC patient population has been reported to be lower compared with the general LSCC population, although the reported follow-up times are relatively short $(2,7)$.

Conventional risk factors such as tobacco smoking have been observed as being less common in young patients with head and neck squamous cell carcinoma, including LSCC $(4,7,9,13)$. This has led to the speculation of other potential etiological factors dominating in this patient group such as immunodeficiency, poor oral hygiene (10), genomic instability (4, 14), reflux, Helicobacter pylori infection (15), and 
occupational carcinogens (4). Studies investigating HPV as a potential risk factor have also emerged reporting an HPV incidence of 7.4 to $75 \%$ in LSCC samples (16, 17). However, no causative role for HPV has been identified in $\operatorname{LSCC}(16,17)$.

The present study is a survey of the nationwide Finnish Cancer Registry (FCR) data for all patients diagnosed with LSCC at the age of 40 years or under in Finland during 1953-2012 $(n=151)$ representing one of the largest series on this topic $(2,6-10,13)$. In addition, an institutional series of patients treated at the Helsinki University Hospital (HUH) (n=35) during the years 1967-2014 is reviewed, to shed light on comorbidities and lifestyle factors, which were not available in the entire FCR. Our primary aim was to investigate the incidence, possible etiological factors, survival and subsequent cancer events (recurrences and SPCs) in young patients with LSCC. 


\section{Materials and Methods}

Patient selection flow for the study is presented in Figure 1.

\section{Patient data - the Finnish Cancer Registry}

For nationwide epidemiological analysis, data on all patients diagnosed with LSCC at the age of 40 years or under in Finland during 1953 to 2012 were collected from the Finnish Cancer Registry (FCR) and reviewed. Data on follow-up time, cause of death and possible SPCs were available for all patients. Stage and grade of the tumors, as well as the given treatment, were more heterogeneously reported, and thus unavailable for comparative analyses.

\section{Patient data - institutional series, Helsinki University Hospital (HUH)}

Clinical data on all patients who had been diagnosed with LSCC at the age of 40 or under, during the period between the $1^{\text {st }}$ January 1967 and $31^{\text {st }}$ December 2013 at the HUH, Helsinki, Finland, were retrospectively reviewed. Data on this institutional patient population were retrieved to supplement the lacking information in the FCR data regarding etiological factors and LSCC recurrence. A total of 35 patients at the age of 40 years or under were diagnosed and treated for LSCC at HUH.

The hospital records were reviewed for patient demographics including possible etiological factors, such as smoking, drinking and comorbidities as well as LSCC recurrence.

\section{Statistical analyses}

Statistical analyses were conducted with the SPSS statistical package (version 20, IBM corporation, USA). Survival outcomes were calculated with the Kaplan-Meier 
method from the diagnosis to the first event. For overall survival (OS), death regardless of cause was used as an event. For disease-specific survival (DSS), death from LSCC was used as an event.

\section{Ethical considerations}

An institutional research permission was granted for the study protocol. Due to the study design (retrospective chart review) formal Research Ethics Board approval was not warranted, in accordance with the Finnish legislation. 
Results

\section{Finnish Cancer Registry data}

There were 151 patients identified from the FCR. Patient and tumor characteristics are shown in Table 1 . The incidence of LSCC in this age group was $0.1 / 100$ 000/year in men and 0.01/100 000/year in women. The mean follow-up time was 21 years (median 21; range, 0.3-59). The treatment of patients according to tumor stage is summarized in Table 2. The 5- and 10-year overall survival (OS) was $84 \%$ and $75 \%$, respectively (Figure 2). The 5- and 10-year disease-specific survival (DSS) was $88 \%$ and $84 \%$, respectively (Figure 3). The 5- and 10-year OS and DSS, stratified by tumor localization and stage, are presented in Table 3. The treatment outcome for Stage 0-II patients and supraglottic primary location patients was excellent, with 5and 10-year DSS figures over 90\%. For Stage III-IV and supraglottic LSCC patients the figures were significantly lower.

Thirty-nine (26\%) of the patients developed SPCs. The mean time from the diagnosis of LSCC to the diagnosis of the SPC was 28 years (median, 28; range, 6-45). The localizations of SPCs are shown in Table 3 and their incidence according to follow-up time in Figure 4. The most common sites for SPCs were the lungs and trachea (14 patients; $36 \%$ of all SPCs). Five patients presented with further primary tumor(s) after the SPC (3\% of all patients, $13 \%$ of patients with SPCs).

\section{Helsinki University Hospital (HUH) institutional data}

Thirty-five young LSCC patients were treated at the HUH during 1967-2014, of whom seven $(20 \%)$ were female and $28(80 \%)$ male. The age range of the patients was $18-40$ years. Of the 35 patients with adequate information on smoking, only six (17\%) had never smoked. For $15(43 \%)$ patients' information on the duration of 
smoking was available with a mean of 19 years until the diagnosis (range, 3-33). Unfortunately, for the majority of patients $(n=28,80 \%)$ there was no information on alcohol consumption. None of our patients had had an earlier malignant tumor nor had they received earlier radiotherapy in the head and neck area. Five (14\%) patients had a family history of a malignant tumor, and one of these was in the head and neck area (no further details were available). Psychiatric conditions were reported in three (9\%) patients, other medical conditions (such as high blood pressure) were expressed in single patients.

In 31 (89\%) patients the tumor was histologically classified as squamous cell carcinoma and four (11\%) had a variant of squamous cell carcinoma (e.g. fusocellular carcinoma and papillomatous carcinoma). The mean follow-up time until death or the last follow-up visit was 18 years (median 15 years; range, 8 months -46 years). Ten (28\%) patients experienced LSCC recurrence during follow-up. The median time to recurrence was 13 months (mean 25; range, 4-69 months), with the majority of recurrences occurring within two years of treatment. 


\section{Discussion}

Studies on young patients with LSCC are scarce as the condition is rare. In studies assessing young patients with LSCC, the age limit has commonly been set to 40 years or younger $(2,6,7,9,13)$ and the number of patients in the individual series typically tends to be limited, ranging from 20 to $99(6,7,10)$.This study presents general factors of all patients diagnosed with LSCC at the age of 40 years or under in Finland over a sixty-year period. Tumor stage and subsite distribution did not differ significantly from those previously reported for the general LSCC population in Finland (1).

The survival figures of the young patients with LSCC did not differ from the general LSCC population in Finland, as is a similar finding in some other studies comparing the young and general cohorts $(6,9)$, although others report a favorable survival in younger patients $(7,8)$. The 5-year DSS was $88 \%$ in the current study and $80 \%$ in the data recently published for Finnish LSCC patients in general(1). In a study by Nachalon et al., the reported 5-year survival rate was 69\%, significantly lower than that observed in the control group of older patients (80\%) (9). However, Stage III-IV disease was predominant in their young patient group (62\%), which is in contrast to our 151 FCR patients having predominantly Stage 0-II disease (66\%). When stratifying their survival outcomes according to stage, they observed no differences in survival between the young and older patients.

The reported rate of disease persistence or recurrence in LSCC is up to $30 \%(11,12)$. This corresponds well with the recurrence rate $(28 \%)$ in the current material of young patients treated at the HUH. Furthermore, the majority of recurrences in patients with 
LSCC tend to occur during the first two years $(12,18)$. In this material, all locoregional recurrences occurred during the first six years.

Patients with LSCC have been reported to be at an increased risk for SPCs, the risk varying from 1.68 to 1.92 -fold reported on population level $(3,19)$. Our analysis of the SPCs with a mean follow-up of 21 years indicates that the majority of SPCs in this population occur relatively late, i.e. several decades after the primary LSCC diagnosis. This differs from the occurrence of SPCs in the general population of LSCC patients, in whom they start occurring already during the first decade after LSCC diagnosis. Milano et al. reported the incidence of second primary lung cancer after LSCC to be $6 \%, 11 \%$, and $16 \%$ at 5,10 , and 15 years, respectively (20). The study of Albright et al., with a follow-up time limited to 15 years, reported LSCC patients under the age of 40 to have a lower risk for SPCs compared to older patients ( $8 \%$ vs $21 \%$ ) (2). We, too, noted an almost complete absence of SPCs during these first 15 years of follow-up. However, with an extended follow-up up to 59 years with a median of 21 years, SPCs occurred in $26 \%$ of patients, an occurrence rate comparable to the LSCC population in general (11). Young LSCC patients seem to develop SPCs at a similar age to the general LSCC population, which is only recognized with extended follow-up. Similar to Morris et al., the most common sites for SPCs were lung or trachea, comprising $36 \%$ of all (19). The majority of the SPCs in our cohort patients presented in the lungs concurrent with other published series (3, 19). Although it is not possible to assess the effect of life-style factors based on our data, the presentation age and location of the SPCs in our cohort of young LSCC patients may indicate the presence of a cumulative effect of carcinogenic agents. 
Risk factor assessment in our data was only possible for patients in the institutional series. Smoking was common among our patients $(83 \%$ of the patients with information), but our data lacked adequate information on alcohol consumption. Nachalon et al. reported a somewhat lower percentage of smokers $(38 \%)$ in their series of 13 young LSCC patients (9). The comorbidities of our young patients were sparse and insignificant: none of our patients had a history of congenital or acquired immune deficiency, and neither a history of Helicobacter pylori infection or nor papillomatosis which are possible etiological risk factors discussed by other authors in the literature $(10,15,17)$. HPV status had not been assessed in any of the tumors in the current material due to the expected low frequency of HPV positivity in LSCC (16). More recent studies show higher rates of HPV in the general LSCC population, perhaps reflecting a rise in HPV incidence (16). There have been speculations about the genetic vulnerability of young patients with head and neck cancer for malignant disease development (13). None of the 35 patients had family members with LSCC or any other cancers, except for one patient who had a parent with an unspecified tumor in the head and neck area. Ultimately, based on these limited data, we were not able to identify any clear distinction between younger and older patients with LSCC with regards to etiological factors.

The main strengths of this study are the comprehensive, large cohort of patients and the extended follow-up time provided by the FCR, which enables a more thorough analysis of SPCs compared with most published studies. Our study suffers a few shortcomings. The retrospective nature of this study is a clear limitation. Although the institutional data allowed us to estimate the possible significance of smoking and comorbidities in the etiology of LSCC in young patients, the data regarding lifestyle 
factors were non-comprehensive and incohesive. This hindered the proper assessment of their effect on the development of not only primary LSCC but also SPCs. However, due to the rareness of LSCC in the young, prospective studies in this patient group aiming to report SPCs are not feasible.

\section{Conclusions}

Location, treatment and outcome of these tumors among young patients did not differ from those presented in the literature for the general LSCC patient population. There appears to be no rationale for treating young patients differently. Despite the abnormally early onset of their laryngeal cancers, SPCs in young patients occurred approximately at the same age as in LSCC patients in general. The interval to SPCs was thus considerably longer compared with the LSCC population in general. Potential factors underlying this phenomenon could not be elucidated in this retrospective study and remain as an interesting research area.

\section{Disclosure statement}

The authors report no conflict of interest. 


\section{References}

1. Haapaniemi A, Koivunen P, Saarilahti K, Kinnunen I, Laranne J, Aaltonen LM, et al. Laryngeal cancer in Finland: A 5-year follow-up study of 366 patients. Head \& neck. 2014.

2. Albright JT, Karpati R, Topham AK, Spiegel JR, Sataloff RT. Second malignant neoplasms in patients under 40 years of age with laryngeal cancer. Laryngoscope. 2001;111(4 Pt 1):563-7.

3. Gao X, Fisher SG, Mohideen N, Emami B. Second primary cancers in patients with laryngeal cancer: a population-based study. Int J Radiat Oncol Biol Phys. 2003;56(2):427-35.

4. Bradley PJ. Laryngeal cancer in nondrinker nonsmoker young patients: a distinct pathological entity? Curr Opin Otolaryngol Head Neck Surg. 2016;24(2):140-7.

5. Talamini R, Bosetti C, La Vecchia C, Dal Maso L, Levi F, Bidoli E, et al. Combined effect of tobacco and alcohol on laryngeal cancer risk: a case-control study. Cancer causes \& control : CCC. 2002;13(10):957-64.

6. Shvero J, Hadar T, Segal K, Abraham A, Sidi J. Laryngeal carcinoma in patients 40 years of age and younger. Cancer. 1987;60(12):3092-5.

7. Lacy PD, Piccirillo JF, Merritt MG, Zequeira MR. Head and neck squamous cell carcinoma: better to be young. Otolaryngol Head Neck Surg. 2000;122(2):253-8.

8. Misono S, Marmor S, Yueh B, Virnig BA. Treatment and survival in 10,429 patients with localized laryngeal cancer: a population-based analysis. Cancer. 2014;120(12):1810-7.

9. Nachalon Y, Alkan U, Shvero J, Yaniv D, Shkedy Y, Limon D, et al. Assessment of laryngeal cancer in patients younger than 40 years. Laryngoscope. 2018;128(7):1602-5.

10. Rutt AL, Hawkshaw MJ, Sataloff RT. Laryngeal cancer in patients younger than 30 years: a review of 99 cases. Ear Nose Throat J. 2010;89(4):189-92.

11. Brandstorp-Boesen J, Sorum Falk R, Folkvard Evensen J, Boysen M, Brondbo K. Risk of Recurrence in Laryngeal Cancer. PLoS One.

2016;11(10):e0164068.

12. Francis DO, Yueh B, Weymuller EA, Jr., Merati AL. Impact of surveillance on survival after laryngeal cancer in the medicare population. The Laryngoscope. 2009;119(12):2337-44.

13. Wang J, Zhao X, Pan X, Zhao L, Zhou J, Ji M. The role of primary surgical treatment in young patients with squamous cell carcinoma of the larynx: a 20 year review of 34 cases. World J Surg Oncol. 2015;13:283.

14. Wang Y, Irish J, MacMillan C, Brown D, Xuan Y, Boyington C, et al. High frequency of microsatellite instability in young patients with head-and-neck squamous-cell carcinoma: lack of involvement of the mismatch repair genes hMLH1 AND hMSH2. Int J Cancer. 2001;93(3):353-60.

15. Zhou J, Zhang D, Yang Y, Zhou L, Tao L. Association between helicobacter pylori infection and carcinoma of the larynx or pharynx. Head Neck. 2016;38 Suppl 1:E2291-6.

16. Torrente MC, Rodrigo JP, Haigentz M, Jr., Dikkers FG, Rinaldo A, Takes RP, et al. Human papillomavirus infections in laryngeal cancer. Head Neck.

2011;33(4):581-6. 
17. Duray A, Descamps G, Arafa M, Decaestecker C, Remmelink M, Sirtaine N, et al. High incidence of high-risk HPV in benign and malignant lesions of the larynx. Int J Oncol. 2011;39(1):51-9.

18. Lyhne NM, Johansen J, Kristensen CA, Andersen E, Primdahl H, Andersen LJ, et al. Pattern of failure in 5001 patients treated for glottic squamous cell carcinoma with curative intent - A population based study from the DAHANCA group. Radiother Oncol. 2016;118(2):257-66.

19. Morris LG, Sikora AG, Patel SG, Hayes RB, Ganly I. Second primary cancers after an index head and neck cancer: subsite-specific trends in the era of human papillomavirus-associated oropharyngeal cancer. J Clin Oncol. 2011;29(6):73946.

20. Milano MT, Peterson CR, 3rd, Zhang H, Singh DP, Chen Y. Second primary lung cancer after head and neck squamous cell cancer: population-based study of risk factors. Head \& neck. 2012;34(12):1782-8. 


\section{Figure legends}

Figure 1. Selection of study populations: the Finnish Cancer Registry data and the Helsinki University Hospital data.

Figure 2. Overall survival after diagnosis in patients 40 years of age or younger diagnosed with laryngeal squamous cell carcinoma in Finland during 1953-2012 $(n=151)$.

Figure 3. Disease-specific survival after diagnosis in patients 40 years of age or younger diagnosed with laryngeal squamous cell carcinoma in Finland during 1953-2012 (n=151).

Figure 4. Cumulative incidence of second primary cancers $(n=39)$ in patients 40 years of age or younger diagnosed with primary laryngeal squamous cell carcinoma $(\mathrm{n}=151)$. 